\title{
Rapid-deployment valves: Finally the fog is lifting-benefits beyond crossclamp and bypass times
}

\author{
Günther Laufer, MD, PhD, ${ }^{a}$ Dominik Wiedemann, $\mathrm{MD},{ }^{\mathrm{a}}$ and Walter Randolph Chitwood, Jr, $\mathrm{MD}^{\mathrm{b}}$
}

\footnotetext{
From the ${ }^{\mathrm{a}}$ Department of Cardiac Surgery, Medical University of Vienna, Vienna, Austria; and ${ }^{\mathrm{b}}$ East Carolina Heart Institute, East Carolina University, Greenville, NC.

Received for publication April 11, 2017; revisions received June 9, 2017; accepted for publication June 26, 2017; available ahead of print September 6, 2017.

Address for reprints: Günther Laufer, MD, PhD, Department of Cardiac Surgery, Medical University of Vienna, Währinger Gürtel 18-20, Vienna 1090, Austria (E-mail: guenther.laufer@meduniwien.ac.at).

J Thorac Cardiovasc Surg 2017;154:1527-31

0022-5223/\$36.00

Copyright (C) 2017 by The American Association for Thoracic Surgery

http://dx.doi.org/10.1016/j.jtcvs.2017.06.065
}

Cardiac surgeons now face the endless innovative drive toward transcatheter aortic valve implantation or transcatheter aortic valve replacement (TAVR). Thus, we are challenged to continue to improve the early, midterm, and long-term outcomes of surgical aortic valve replacement (SVAR). The key elements to achieve this goal are minimizing surgical access and embracing new surgical valve technologies. Recent efforts to improve surgical aortic valve prostheses have been focused on sutureless surgical aortic valve replacement (SU-SAVR) or rapid-deployment surgical aortic valve replacement (RD-SAVR). Two sutureless prostheses, the $3 \mathrm{f}$ Enable (Medtronic Inc, Minneapolis, Minn) and Perceval valves (LivaNova PLC, London, United Kingdom), were the first ones to obtain a Conformité Européenne mark in 2009 and 2011, respectively. However, the $3 f$ Enable valve was withdrawn from the market in 2015. In contrast to these sutureless, nitinol-based valves, the Intuity valve system (Edwards Lifesciences LLC, Irvine, Calif) is constructed on the long-term proven Magna Ease Perimount (pericardial) aortic valve platform (Figure 1). It has a quick-connect, stent-based (Figure 2) deployment system that requires only 3 guiding sutures that are tied down after implantation (Figure 3, Video 1). Thus, it is not considered to be a pure sutureless prosthesis but rather a rapid-deployment valve. The striking fundamental design of Intuity differentiates and suggests that rapid deployment does not necessarily mean sutureless! The term "rapid-deployment valve" refers in the context of this article only to the Edwards Intuity valve. In a general sense, any sutureless valve (Perceval, Enable) could be considered a rapid-deployment valve because implantation is quickly facilitated. Considering recently published data from both multicenter and single institution Intuity valve trials, we now should ask the question: Has any substantial progress been made?

Reports of early outcomes have shown a consistent reduction in aortic crossclamp and cardiopulmonary bypass (CPB) times ${ }^{1-4}$ with this valve. Borger and colleagues ${ }^{2}$ reported a randomized comparison of the Intuity valve, delivered via an upper hemi-sternotomy, with a conventional

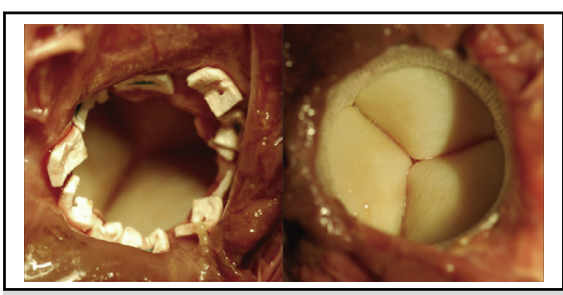

Perimount Magna Ease valve (left) and Intuity valve (right) (Edwards Lifesciences LLC, Irvine, Calif) from the ventricular aspect.

Central Message

Rapid-deployment aortic bioprostheses may provide an improved safety and efficacy profile compared with conventional surgical aortic bioprosthesis and transcatheter aortic valves.

See Editorial Commentary page 1532.

bioprosthesis implanted through a full sternotomy. They showed a highly significant reduction in myocardial ischemic time (54.0 minutes and 41.3 minutes, respectively), favoring the Intuity valve despite the more challenging minimally invasive surgical access. These findings were confirmed in both the European TRITON (ClinicalTrials.gov Identifier: NCT01445171) $^{1,3,5}$ and the recently published US TRANSFORM (ClinicalTrials.gov Identifier: PNCT01700439) trials. ${ }^{4}$ Both trials documented a remarkable reduction in crossclamp and CPB times for both isolated sternotomy-based and minimally invasive RD-SAVR operations when compared with Society of Thoracic Surgeons (STS) National Database values. Even more important, these times were reduced significantly in combined cardiac procedures. Likewise, similar impressive reductions of crossclamp and CPB times have been demonstrated for SU-SAVR with the Perceval valve. ${ }^{6}$

In both TRITON and TRANSFORM trials, each involved the surgeon implanting the Intuity valve for the first time. Thus, without the learning curve effect, crossclamp and CPB times might have been even shorter. With regard to shortened crossclamp and CPB times, this factor may offset

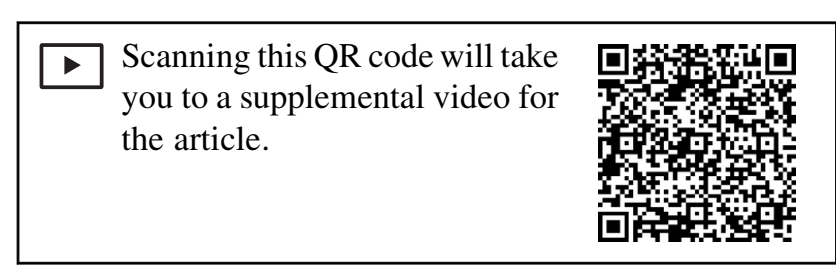




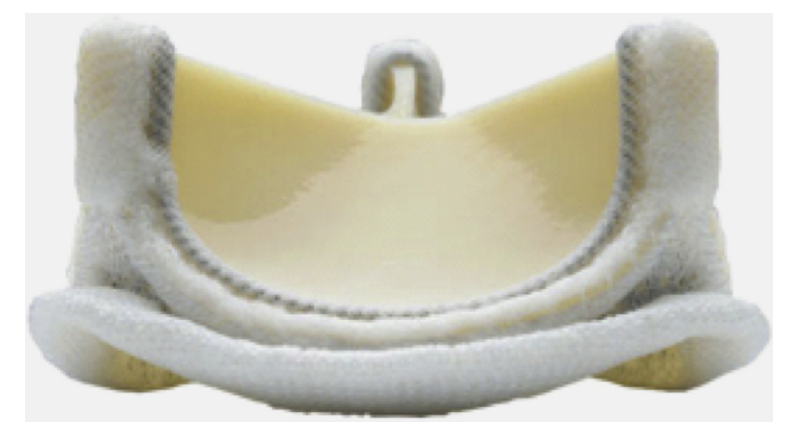

FIGURE 1. Front view of an Edwards Perimount Magna Ease pericardial valve. (Edwards Lifesciences LLC, Irvine, Calif).

the possible selection bias of including only experienced centers in these studies and makes the comparison with STS database values even more reasonable. These benefits are impressive and may suggest a positive impact on hard clinical end points (eg, 30-day mortality). The 30-day mortality in the TRITON and TRANSFORM studies was $1.7 \%$ and $0.9 \%$, respectively. This appears to be noticeably low, considering the advanced clinical profiles of patients in both studies (mean age, $75 / 73$ years; only $55 / 62 \%$ isolated RD-SAVR; mean STS score, 3.5/2.5, respectively). Although multiple factors, short crossclamp, and CPB times, among others, might have contributed to this favorable early mortality, the multi-institutional international experience ( 35 centers) underscores the reproducibility of these excellent early outcomes.

Additional benefits of RD-SAVR may be subtle and require more detailed, accurate scrutiny to reveal them. In this regard, assessment of hemodynamic performance by echocardiography is particularly sensitive to interobserver differences. Highly standardized, independent core laboratory measurements provide the most reliable information. We emphasize that data from both the TRITON and TRANSFORM trials were based on this methodology and should be viewed to be more reliable than echocardiographic data from retrospective reports from single centers.

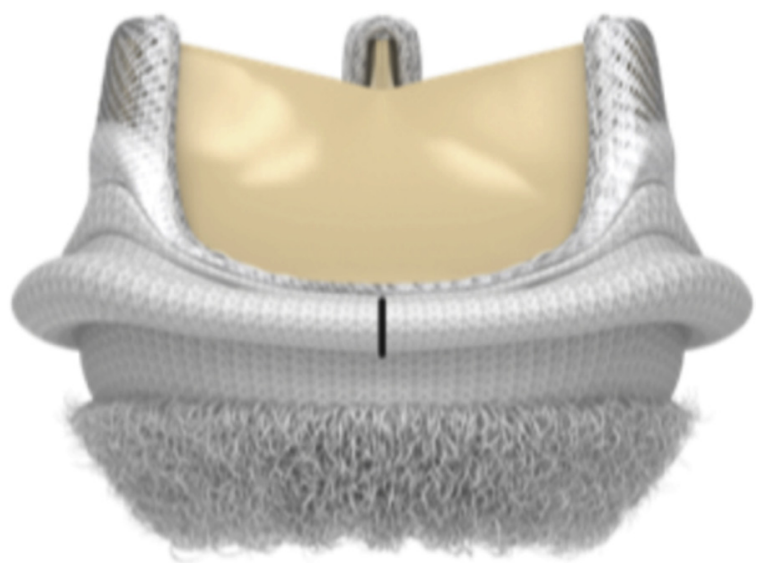

FIGURE 2. Front view on an undeployed Edwards Intuity Elite rapiddeployment pericardial valve.

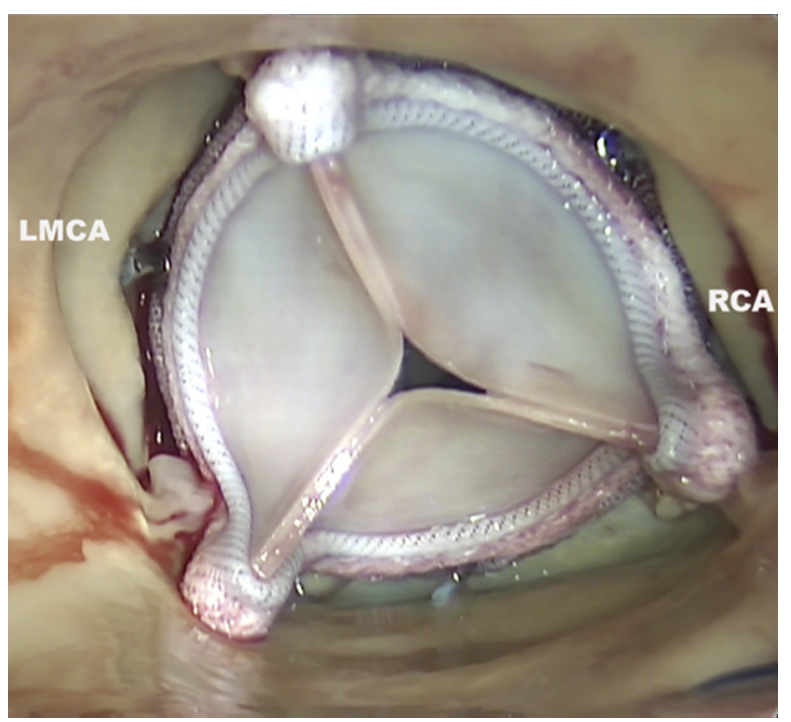

FIGURE 3. Aortic aspect of a fully implanted rapid-deployment Intuity valve with leaflets closed. The 3 guiding sutures at the nadir have been tied down by a COR-KNOT device (LSI Solutions Inc, Victor, NY). $L M C A$, Left main coronary artery; $R C A$, right coronary artery.

The Intuity valve system design not only enables quicker and easier implantation but also promises improved hemodynamic performance. With the use of in vitro testing, Capelli and colleagues ${ }^{7}$ demonstrated that pledget-armed sutures adversely affect the hemodynamic performance of biologic aortic valves when compared with the stentbased anchoring system of the Intuity system (Central Image). Both TRITON and TRANSFORM trials now confirm these previously postulated advantages through highly reliable clinical data: 1-year mean gradients of $9.0 \pm 3.5 \mathrm{~mm}$ $\mathrm{Hg}$ and $10.3 \pm 3.8 \mathrm{~mm} \mathrm{Hg}$ and effective orifice areas of $1.7 \pm 0.2 \mathrm{~cm}^{2}$ and $1.7 \pm 0.2 \mathrm{~cm}^{2}$, respectively. ${ }^{4,5}$ This hemodynamic performance, which was present in all implanted valve sizes, seems to be superior to most conventional sewn-in bioprostheses. ${ }^{8,9}$ Similar gradient improvements have been shown for newer-generation conventional pericardial valves in which the leaflet tissue is mounted on the stent-frame exterior (Trifecta, St Jude Medical, St Paul, Minn ${ }^{10}$; Mitroflow, Sorin Group, Saluggia, Italy $^{11}$ ), as well as the sutureless Perceval bioprosthesis. ${ }^{12,13}$ The long-term durability of these newer valves remains to be determined.

In the TRITON trial, optimal hemodynamic performance was shown for the Intuity valve by the unequivocally and exceptionally low rate of severe patient-prosthesis mismatch (PPM) from discharge to 3 years $(2.4 \%$ $3.9 \%))^{5,14}$ Theron and colleagues ${ }^{15}$ focused on the small aortic root (only 19-mm and 21-mm Intuity valves) and reported a severe PPM rate as low as $15 \%$ in this subset. This is remarkable because severe PPM rates with conventional bioprosthesis SAVR have been approximately $20 \%$ according to a recent meta-analysis by Takagi and colleagues. ${ }^{16}$ 


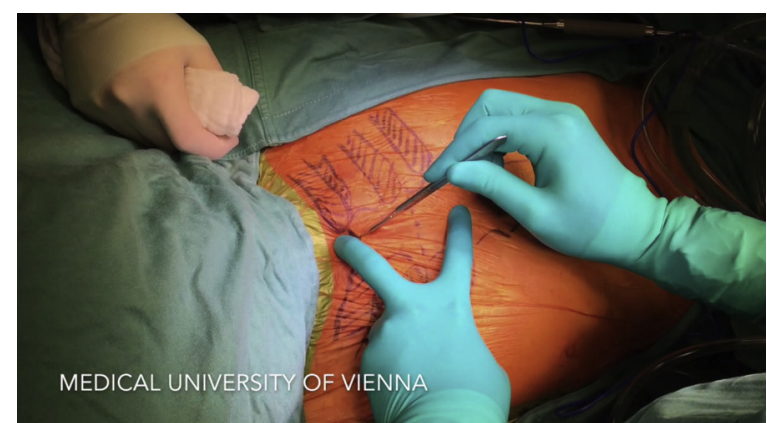

VIDEO 1. Isolated RD-SAVR for severe aortic stenosis via less-invasive upper hemisternotomy access. Video available at: http://www.jtcvsonline. org/article/S0022-5223(17)31583-0/fulltext.

In addition, these excellent data deserve an indirect comparison with severe PPM following TAVR. In the same metaanalysis, Takagi and colleagues ${ }^{16}$ showed an overall early (discharge and 3 months) severe PPM rate of $8 \%$ for TAVR. Comparison of data suggests not only a potential PPM disadvantage for conventional SAVR versus TAVR but also a possible significant advantage of RD-SAVR over SAVR and TAVR. Accordingly, these results and those for both Perceval $\left(0 \%\right.$ severe $\left.\mathrm{PPM}^{6}\right)$ and Trifecta valve studies support the notion that future hemodynamic performance of TAVR should not be compared with conventional SAVR data anymore.

Low transvalvular gradients of this valve are attributed to both the absence of subvalvular suture pledgets and the funnel-shaped expanded stent frame, which conveys a smooth transition along the left ventricular outflow tract and across the prosthesis (Figure 4). Johnston and colleagues ${ }^{17}$ demonstrated that severe PPM and elevated mean transvalvular gradients were associated strongly

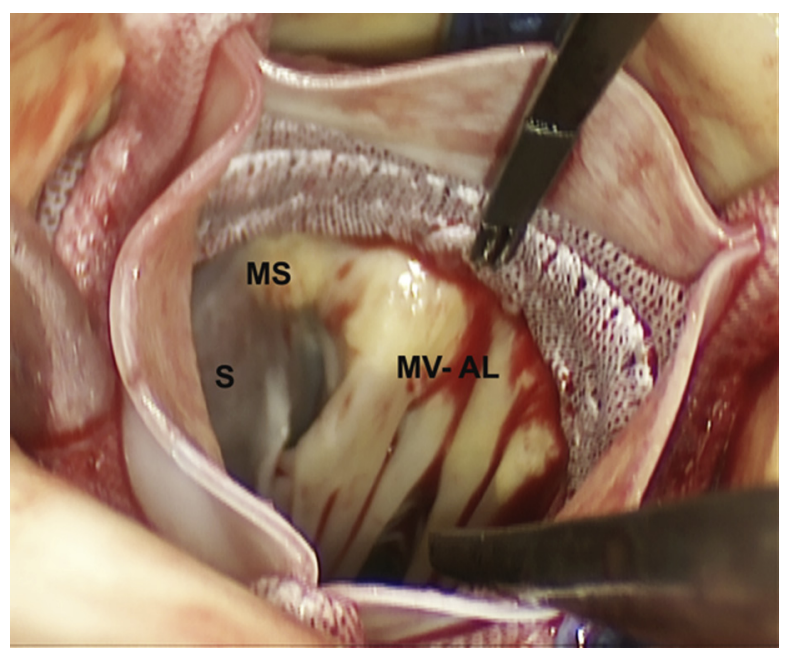

FIGURE 4. Subvalvular aspect of a fully implanted rapid-deployment Intuity valve through opened leaflets. The expanded stent frame is smoothly aligned with the left ventricular outflow tract. $M S$, Membranous septum; $S$, muscular septum; $M V-A L$, anterior leaflet of the mitral valve. with accelerated SVD in 12,569 Perimount valve implants. Hopefully, lower gradients with the Intuity design will contribute to reduced future SVD. Accurate follow-up beyond 5 years is most important for the TRITON and TRANSFORM trials to test this hypothesis and unveil this postulated beneficial effect to minimize SVD.

RD-SAVRs show a remarkable resistance to prosthetic valve endocarditis (PVE). In the TRITON trial, the early incidence was $0.2 \%$ (late $0.2 \%$ /per patient-year), and in TRANSFORM there were no early cases or throughout late follow-up. Recently published endocarditis data from a TAVR registry showed a higher rate at $1.1 \% /$ per patient-year (95\% confidence interval, 1.1-1.4) ${ }^{18}$ which suggests a worse outcome for this objective performance criterion compared with RD-SAVR. The significance of this indirect comparison could be questionable but hypothesis generating at the same time. By deductive reasoning, it is plausible that this salutary effect really exists from a number of factors, which may include the lack of crimping trauma, complete removal of calcified and necrotic material during implantation, low paravalvular leak rates that have been associated with subsequent PVE (at least in $\mathrm{TAVR}^{18}$ ), and higher sterility levels in an operating room versus a catheterization suite. When compared with conventional SAVR, the exceptionally low PVE rates with RDSAVR may be explained by the covered stent smooth surface, which reduces surface roughness that is present with multiple pledgets in the outflow tract (Central Image). Last, the valve can be implanted with almost no contact between the surgeon's gloves and the prosthesis, especially if a knot pusher is used to tie the guiding sutures. On the basis of these considerations, there is a high likelihood that very low PVE in RD-SAVR really exists, and further clinical data are pivotal to confirm these trial findings.

Valve thrombosis in TRITON and TRANSFORM patients $(\mathrm{n}=1126)$ was absent at both early and late clinical follow-ups. A report by Makkar and colleagues ${ }^{19}$ emphasized the potential significance of early subclinical bioprosthesis valve thrombosis (BPVT). This phenomenon seems to be underreported in previous studies and occurs more frequently after TAVR than conventional SAVR. Most recently, it was confirmed to be highly significant in favor of SAVR versus TAVR with $4 \%$ versus $13 \%$, respectively. ${ }^{20}$ Most studies did neither serial transthoracic echocardiography (TTE) examinations within the first year nor more sophisticated investigations such as transesophageal echocardiography or 4-dimensional volume-rendered computed tomography scans. Thus, underreporting of BPVT in TRITON and TRANSFORM cannot be excluded because subclinical valve thrombosis often is associated with absent or minimal gradient increases. Moreover, abnormal leaflet motion and thickening are difficult to detect by TTE. However, in the TRITON trial, the lack of BPVT evidence on high-quality, 
core laboratory-adjudicated TTE images $(\mathrm{N}=1126$ patients) at 1 year and 5 years and gradient stability suggest that it is unlikely. ${ }^{5}$ Nevertheless, a prospective study using 4-dimensional volume-rendered computed tomography scans with different antithrombotic strategies would add valuable information.

Therefore, is there only good news? Certainly, costs of the implant are higher compared with standard prostheses, but general economic outcome end points have been documented to be equal or even superior. ${ }^{21}$ Almost all aspects of efficacy and safety parameters of RD-SAVR are better or equal to conventional SAVR or TAVR. The anchoring mechanism design of the Intuity valve is based on balloon inflation of a subvalvular stent frame with radial pressure apposition throughout the distal subvalvular outflow tract. Theoretically, any pressure below the leaflet attachment zone induces the risk of a cardiac conduction disturbance. This drawback has been observed even in the most recent versions of TAVR valves, ${ }^{22,23}$ in which the new early permanent pacemaker implantations (PPIs) are approximately $20 \%$. With SAVR, the prosthesis implantation mechanical stress is restricted to the leaflet attachment zone and immediately below where the sutures are placed. Early new PPI rates with conventional SAVR have been shown to be as low as $3.0 \%$ for an isolated SAVR but as high as $11.2 \%$ when combined with another procedure. ${ }^{24}$ In both TRITON and TRANSFORM trials, RD-SAVR was performed in patients with the higher-risk profile of a combined cardiac operation in $45.9 \%$ and $37.5 \%$, respectively. Thus, a higher likelihood for new conduction disturbances existed preoperatively. Although European TRITON data showed an early PPI rate of $6.9 \%$, the US TRANSFORM trial revealed a higher early PPI rate $(14.0 \%$ overall and $6.0 \%$ without previous conduction abnormalities).

These observed differences in PPIs have to be interpreted with caution: First, the implant technique, although the valve is fixed at the nadir in all 3 sinuses, allows variability as to how deep the valve descends in the outflow tract. If commissural suspension sutures are used for better exposure and traction is exerted during introduction of the valve, the stent frame can be deployed deeper into the conduction sensitive area in the right nonsubcommissural triangle, promoting a higher risk of new conduction disturbances. In comparison, implant technique details also seem to affect PPI after Perceval SU-SAVR with a recently reported high $23 \%$ rate $^{25}$ and lower $11.6 \%{ }^{13}$ rate, which is obviously affected by the use of an inflation balloon for implantation. Second, sizing is critical. Surgeons in the "learning phase" of implantation may tend to oversize the valve to avoid paravalvular leak, which can result in increased radial stress along the subvalvular rim. Third, with this valve, approximately $30 \%$ of new conduction disturbances revert within 1 week postoperatively. Generally, in the United
States an earlier patient discharge strategy is present than in European centers. Thus, in TRANSFORM more patients may have had pacemakers implanted early to ensure safety at discharge. A watchful waiting strategy should allow more patients to recover from transitory rhythm disturbances and result in a lower PPI rate. Last, use of antiarrhythmic drugs (eg, beta-blockers, amiodarone) could contribute to observed differences in trans-Atlantic PPIs. These are speculative arguments, and more data are needed to confirm the hypothesis that the aforementioned factors play an important role in new PPI rates. The most recent European Intuity prospective registries (FOUNDATION ClinicalTrials.gov Identifier: NCT02338154 trial, presented at the 96th American Association for Thoracic Surgery annual meeting, May 14-18, 2016, Baltimore, Md, completed $\mathrm{n}=493$ and MISSION ClinicalTrials.gov Identifier: NCT02907463 trial, recruitment ongoing) should provide more insight into the question of PPI. Moreover, we anticipate from these studies additional information on the incidence and prognostic implications of a newly developed left bundle branch block, which is an end point that thus far has not been reported in other RD-SAVR multicenter trials.

Today, the fog around RD-SAVR prosthesis implantation is lifting beyond the benefits of reduced crossclamp and CPB times, as well as facilitation of minimally invasive access. The advances in design have been translated into subtle but clinically significant improvements that make RDSAVR fully competitive, although we are inclined to say superior, to TAVR. Because many of these benefits are subtle, accurate investigations with meticulous data collection beyond 5 years are needed to consummate these salutary findings. Careful distinction between RD-SAVR and SU-SAVR is mandated for outcome evaluations because both valve types differ substantially in design and implant technique.

\section{Conflict of Interest Statement}

G.L.: consultant, Edwards Lifesciences. D.W.: speaker honorarium, Edwards Lifesciences. W.R.C.: consultant Neochord, Inc, Co, and Principal Investigator, Edwards Transform Clinical Trial.

\section{References}

1. Kocher AA, Laufer G, Haverich A, Shresta M, Walther T, Misfeld M, et al. One-year outcomes of the Surgical Treatment of Aortic Stenosis With a Next Generation Surgical Aortic Valve (TRITON) trial: a prospective multicenter study of rapid-deployment aortic valve replacement with the EDWARDS INTUITY Valve System. J Thorac Cardiovasc Surg. 2013;145:110-6.

2. Borger MA, Moustafine V, Conradi L, Knosalla C, Richter M, Merk DR, et al. A randomized multicenter trial of minimally invasive rapid deployment versus conventional full sternotomy aortic valve replacement. Ann Thorac Surg. 2015; 99:17-25.

3. Wahlers TC, Haverich A, Borger MA, Shresta M, Kocher AA, Walther T, et al. Early outcomes after isolated aortic valve replacement with rapid deployment aortic valve. J Thorac Cardiovasc Surg. 2016;151:1639-47.

4. Barnhart GR, Accola KD, Grossi EA, Woo YJ, Mumtaz MA, Sabik JF, et al. TRANSFORM (Multicenter Experience With Rapid Deployment Edwards 
INTUITY Valve System for Aortic Valve Replacement) US clinical trial: Performance of a rapid deployment aortic valve. J Thorac Cardiovasc Surg. 2017; 153:241-51.e242.

5. Laufer G, Haverich A, Andreas M, Mohr FW, Walther T, Shresta M, et al. Long-term outcomes of a rapid deployment aortic valve: data up to 5 years. Eur J Cardiothorac Surg. April 26, 2017 [Epub ahead of print].

6. Belluschi I, Moriggia S, Giacomini A, Del Forno B, Di Sanzo S, Blasio A, et al. Can Perceval sutureless valve reduce the rate of patient-prosthesis mismatch? Eur J Cardiothorac Surg. 2017;51:1093-9.

7. Capelli C, Corsini C, Biscarini D, Ruffini F, Migliavacca F, Kocher A, et al. Pledget-armed sutures affect the haemodynamic performance of biologic aortic valve substitutes: a preliminary experimental and computational study. Cardiovasc Eng Technol. 2017;8:17-29.

8. Thalji NM, Suri RM, Michelena HI, Greason KL, Dearani JA, Daly RC, et al. Do differences in early hemodynamic performance of current generation biologic aortic valves predict outcomes 1 year following surgery? J Thorac Cardiovasc Surg. 2015;149:163-73.e162.

9. Dalmau MJ, Gonzalez-Santos JM, Blazquez JA, Sastre JA, Lopez-Rodriguez J, Bueno M, et al. Hemodynamic performance of the Medtronic Mosaic and Perimount Magna aortic bioprostheses: five-year results of a prospectively randomized study. Eur J Cardiothorac Surg. 2011;39:844-52.

10. Rubens FD, Gee YY, Ngu JM, Chen L, Burwash IG. Effect of aortic pericardial valve choice on outcomes and left ventricular mass regression in patients with left ventricular hypertrophy. J Thorac Cardiovasc Surg. 2016;152:1291-8.e1292.

11. Asch FM, Heimansohn D, Doyle D, Dembitsky W, Ferdinand FD, Swanson J, et al. Mitroflow aortic bioprosthesis 5-year follow-up: North American prospective multicenter study. Ann Thorac Surg. 2012;94:1198-203.

12. Shrestha M, Fischlein T, Meuris B, Flameng W, Carrel T, Madonna F, et al. European multicentre experience with the sutureless Perceval valve: clinical and haemodynamic outcomes up to 5 years in over 700 patients. Eur J Cardiothorac Surg. 2016;49:234-41.

13. Laborde F, Fischlein T, Hakim-Meibodi K, Misfeld M, Carrel T, Zembala M, et al. Clinical and haemodynamic outcomes in 658 patients receiving the Perceval sutureless aortic valve: early results from a prospective European multicentre study (the Cavalier Trial). Eur J Cardiothorac Surg. 2016;49:978-86.

14. Haverich A, Wahlers TC, Borger MA, Shresta M, Kocher AA, Walther T, et al. Three-year hemodynamic performance, left ventricular mass regression, and prosthetic-patient mismatch after rapid deployment aortic valve replacement in 287 patients. J Thorac Cardiovasc Surg. 2014;148:2854-60.

15. Theron A, Gariboldi V, Grisoli D, Jassaud N, Morera P, Lagier D, et al. Rapid deployment of aortic bioprosthesis in elderly patients with small aortic annulus. Ann Thorac Surg. 2016;101:1434-41.

16. Takagi H, Umemoto T; ALICE (All-Literature Investigation of Cardiovascular Evidence) Group. Prosthesis-patient mismatch after transcatheter aortic valve implantation. Ann Thorac Surg. 2016;101:872-80.

17. Johnston DR, Soltesz EG, Vakil N, Rajeswaran J, Roselli EE, Sabik JF III, et al Long-term durability of bioprosthetic aortic valves: implications from 12,569 implants. Ann Thorac Surg. 2015;99:1239-47.

18. Regueiro A, Linke A, Latib A, Ihlemann N, Urena M, Walther T, et al Association between transcatheter aortic valve replacement and subsequent infective endocarditis and in-hospital death. JAMA. 2016;316:1083-92.

19. Makkar RR, Fontana G, Jilaihawi H, Chakravarty T, Kofoed KF, De Backer O et al. Possible subclinical leaflet thrombosis in bioprosthetic aortic valves. N Engl J Med. 2015;373:2015-24.

20. Chakravarty T, Sondergaard L, Friedman J, De Backer O, Berman D, Kofoed KF, et al. Subclinical leaflet thrombosis in surgical and transcatheter bioprosthetic aortic valves: an observational study. Lancet. 2017;389:2383-92.

21. Moore M, Barnhart GR, Chitwood WR Jr, Rizzo JA, Gunnarsson C, Palli SR, et al. The economic value of INTUITY in aortic valve replacement. $J$ Med Econ. 2016;19:1011-7.

22. Jarrett CM. Permanent pacemaker insertion following transcatheter aortic valve replacement: Not infrequent, not benign, and becoming predictable. J Thorac Cardiovasc Surg. 2017;153:1063-4.

23. Noble S, Stortecky S, Heg D, Tueller D, Jeger R, Toggweiler S, et al Comparison of procedural and clinical outcomes with Evolut $\mathrm{R}$ versus Medtronic CoreValve: a Swiss TAVI registry analysis. EuroIntervention. 2017; 12:e2170-6.

24. Matthews IG, Fazal IA, Bates MG, Turley AJ. In patients undergoing aortic valve replacement, what factors predict the requirement for permanent pacemaker implantation? Interact Cardiovasc Thorac Surg. 2011;12:475-9.

25. Bouhout I, Mazine A, Rivard L, Ghoneim A, El-Hamamsy I, Lamarche Y, et al. Conduction disorders after sutureless aortic valve replacement. Ann Thorac Surg. 2017;103:1254-60. 